\title{
Ratio estimators for the Co-efficient of Variation in a Finite Population
}

Archana, V.

Department of Statistics, Mangalore University

Mangalagangothri, Konaje, Karnataka, India

archana_stkı@yahoo.com

\section{K. Aruna Rao}

Department of Statistics, Mangalore University

Mangalagangothri, Konaje, Karnataka, India

arunaraomu@yahoo.com

\section{Summary}

The Co-efficient of variation (C.V) is a relative measure of dispersion and is free from unit of measurement. Hence it is widely used by the scientists in the desciplines of agriculture, biology, economics and environmental science. In this paper three ratio and three product estimators of C.V are proposed in finite population and small sample performance of the estimators is carried out using bivariate normal distribution. In addition to the small sample mean square error, the performance of the estimators are assessed using the criterion i) mean square error ii) coverage probability and iii) average length of the confidence interval. Such type of model based comparison of the estimators of C.V has not been done in the past and thus is more comprehensive in nature. Certain guidelines for the use of ratio/product estimators of C.V are provided for the benefit of the applied researchers.

Keywords: Model based comparison, Co-efficient of variation, Simple random sampling, Ratio/product estimator, Mean square error.

\section{Introduction}

Inference concerning the population co-efficient of variation (C.V) has drawn the attention of the researchers in the last eight decades. It dates back to the work of Mckay, Pearson and Fieller (1932). C.V is a unitless measure and thus has got more applications rather than the variance or the standard deviation. Standard deviation is difficult to interpret compared to the C.V which when expressed in percentages has straight forward interpretations. Perhaps this is the motivation for the pioneering researchers of C.V like those which are cited previously. Coefficient of variation has been used as a poverty index (Sen (1973)). It has got applications in reliability theory, meteorology and process control. For some of these applications see the references cited in Nairy and Rao (2003) and Patel and Shah (2009). Much of the works on C.V assumes that the underlying distribution is normal. For a recent work on C.V of the normal distribution see Mahmouvand and Hassani (2007) and the references cited there in.

The estimation of C.V in finite populations was discussed initially by Das and Tripathi $(1981 \mathrm{a}, \mathrm{b})$. Later on various researchers have attempted the estimation of C.V which include the works of Rajyaguru and Gupta $(2002,2006)$, Tripathi et al. (2002), Patel and Shah (2009), among others. Since then many estimators of finite population C.V have been proposed which include the classical ratio, 
product and regression estimators. Das and Tripathi (1981) proposed a class of ratio estimators which included the classical ratio/product estimators as particular cases. A more general class of ratio estimators was proposed by Tripathi et al. (2002). In this class they used regression/ regression type of estimators for constructing the generalized ratio estimators. In addition to ratio/ product estimators, regression estimators are also members of this class. An optimal estimator belonging to this class was also derived using two real life datasets. They compared the asymptotic relative efficiency of 22 estimators. Although the optimal estimator performs well, the performance of some of the ratio and regression estimators are better than the sample C.V. Patel and Shah (2009) compared the small sample mean square error (MSE) of five estimators of $\mathrm{C} . \mathrm{V}$ which included two members belonging to the previous class. They also arrived at the conclusion that ratio estimators perform well compared to the sample C.V.

Although the class of estimators proposed by Tripathi et al. (2002) includes a large class of estimators, many estimators belonging to this class are the hybrid estimators of ratio/product and regression estimators. The optimum estimator is difficult to compute and does not seem to be widely used by the applied scientists. Even to this day many ratio and regression estimators of population mean and variance are constructed by the various researchers (see Kadilar and Cingi (2006), Shabbir and Gupta (2007) and Singh and Vishwakarma (2008)). The class of naïve ratio/product and regression estimators of C.V (constructed from sample C.V) is large and the applied scientists need guidelines for the proper choice of these estimators. This has motivated us to re-examine the ratio and product estimators of population C.V. We derive the exact expressions for the bias and MSE of these estimators to the order of $O\left(\mathrm{n}^{-1}\right)$ in simple random sampling without replacement (SRSWOR). These expressions are new and exact. The commonly used practice of obtaining the expressions for the bias and MSE under SRSWOR is by multiplying the finite population correction (1-f), $f=$ $\mathrm{n} / \mathrm{N}$ to the relevant expressions under SRSWR. Our expressions for bias and MSE contain additional terms and are more accurate. These expressions agree with the asymptotic expressions given in Tripathi et al. to the order of $O\left(\mathrm{n}^{-1}\right)$ in SRSWR if we ignore certain terms involving $1 / \mathrm{N}$. This acts as a check regarding the accuracy of the derivations.

In the past model based comparison of ratio (product) estimators of C.V has not been undertaken. Under bivariate normal distribution, extensive simulation is carried out to compare the performance of seven ratio/product estimators. One of the measures of efficiency to compare the point estimators is through the comparison of small sample MSE. Confidence interval provides a link between point estimation and testing of hypotheses and gives comprehensive inference regarding a parameter of interest. Thus in the present investigation in addition to the small sample MSE, coverage probability of the confidence interval and average length of the confidence interval are also used to measure the performance of seven estimators which included three ratio and three product estimators. The ratio estimators using the information on the C.V of the auxiliary variable and mean of the auxiliary variable emerges as the best estimator in the 
class of naïve ratio and product estimators. The results arrived in this paper supplements the conclusion arrived at in Tripathi et al. (2002), Patel and Shah (2009) and gives concluding guidance to the applied scientists regarding the choice of ratio and product estimators of C.V.

The organization of the paper is as follows. Section 2 presents the general expressions for bias and MSE of the ratio/product estimators to the order of $O\left(\mathrm{n}^{-1}\right)$. The results are used to derive the bias and MSE of ratio/product estimators of C.V constructed using sample C.V under simple random sampling. Small sample comparison of the ratio/product estimators of C.V are considered in section 3. A brief discussion on the performance of the estimators is presented in section 4 and paper concludes in section 5 .

\section{Bias and MSE of ratio and product estimators.}

Let $\theta_{y}$ be the parameter of interest of the study variable ' $y$ ' and $\eta_{x}$ be the parameter of the auxiliary variable ' $\mathrm{x}$ ' and $\hat{\theta}_{y}$ and $\hat{\eta}_{x}$ denote their unbiased estimators. Then the ratio estimator of $\theta_{y}$ is given by,

$$
\hat{\theta}_{y_{R}}=\frac{\hat{\theta}_{\mathrm{y}}}{\hat{\eta}_{\mathrm{x}}} \times \eta_{x}
$$

The following theorem gives the general expression for the bias and MSE of ratio estimator $\hat{\theta}_{y_{R}}$.

Theorem 2.1: The expression for the bias and MSE of the ratio estimator $\hat{\theta}_{y_{R}}$ to the order of $O\left(\mathrm{n}^{-1}\right)$ is given by,

$$
\begin{aligned}
& B\left(\hat{\theta}_{y_{R}}\right)=\theta_{\mathrm{y}}\left\{\frac{\mathrm{V}\left(\hat{\eta}_{\mathrm{x}}\right)}{\eta_{x}{ }^{2}}-\frac{\operatorname{Cov}\left(\hat{\theta}_{y}, \hat{\eta}_{x}\right)}{\theta_{y} \times \eta_{x}}\right\}+o\left(\frac{1}{n}\right) \\
& M\left(\hat{\theta}_{y_{R}}\right)=\mathrm{V}\left(\hat{\theta}_{\mathrm{y}}\right)-2 R \operatorname{Cov}\left(\hat{\theta}_{y}, \hat{\eta}_{x}\right)+R^{2} V\left(\hat{\eta}_{x}\right)+o\left(\frac{1}{n}\right)
\end{aligned}
$$

Proof: Proof is straight forward using the $\varepsilon$-approach given in Murthy (1977) and is omitted.

These expressions coincide with the expressions given in Murthy when $\theta_{y}=\bar{Y}$ and $\eta_{x}=\bar{X}$. From the expressions for the MSE of the ratio estimator it follows that, the ratio estimator of $\theta_{y}$ is better than the estimator $\hat{\theta}_{y}$ if $R<\frac{2 \operatorname{Cov}\left(\hat{\theta}_{\mathrm{y}}, \hat{\eta}_{x}\right)}{V\left(\hat{\eta}_{x}\right)}$. 
Thus in general it is not always guaranteed that the ratio estimators perform well compared to the estimator $\hat{\theta}_{y}$. Tripathi et al. used regression type estimators of $\theta_{y}$ to propose a general class of ratio estimators of the form $\hat{\theta}_{y} \times\left(\frac{\eta_{x}}{\hat{\eta}_{x}}\right)^{\alpha}$ and obtained optimum estimators in this class. It may be noted that the optimal choice of $\alpha$ is not equal to one in general.

Theorem 2.2: Let the product estimator of $\theta_{y}$ be given by,

$$
\hat{\theta}_{y_{P}}=\frac{\hat{\theta}_{\mathrm{y}}}{\eta_{\mathrm{x}}} \times \hat{\eta}_{x}
$$

then the bias and MSE of $\hat{\theta}_{y_{p}}$ to the order of $O\left(\mathrm{n}^{-1}\right)$ is given by,

$$
\begin{aligned}
& B\left(\hat{\theta}_{y_{p}}\right)=\theta_{y}\left\{\frac{\operatorname{Cov}\left(\hat{\theta}_{y}, \hat{\eta}_{x}\right)}{\theta_{y} \times \eta_{x}}\right\}+o\left(\frac{1}{n}\right) \\
& M\left(\hat{\theta}_{y_{p}}\right)=\mathrm{V}\left(\hat{\theta}_{y}\right)+2 \operatorname{RCov}\left(\hat{\theta}_{y}, \hat{\eta}_{x}\right)+R^{2} V\left(\hat{\eta}_{x}\right)+o\left(\frac{1}{n}\right)
\end{aligned}
$$

Proof: Proof is straight forward and is omitted.

From the expression for the MSE of the product estimator it follows that the product estimator is efficient than the estimator $\hat{\theta}_{y}$ if $R<-\frac{2 \operatorname{Cov}\left(\hat{\theta}_{y}, \hat{\eta}_{x}\right)}{V\left(\hat{\eta}_{x}\right)}$.

When $\theta_{y}=\bar{Y}$ and $\eta_{x}=\bar{X}$ this condition reduces to the condition that the variables ' $x$ ' and ' $y$ ' have to be negatively correlated.

Remark: If $\hat{\theta}_{y}$ and $\hat{\eta}_{x}$ are the estimators whose bias is of the order $O\left(\mathrm{n}^{-1}\right)$, the expressions for bias given in equation (2.2) and (2.5) contain additional terms corresponding to these biases. The MSE remains unchanged.

These expressions for bias and MSE are general expressions for any sampling design.

The aim of this paper is to compare the naïve ratio and product estimators of population C.V of the study variable when $\eta_{x}$ refers to the mean, variance and C.V of the auxiliary variable. Ratio and product estimators are simple to use and are appealing to the applied scientists and this investigation throws light 
regarding the proper choice of ratio/product estimators. We have derived the bias and MSE of the three ratio and three product estimators of population C.V of the study variable to the order of $O\left(\mathrm{n}^{-1}\right)$ in SRSWR and SRSWOR. The expression for bias to the order of $O\left(\mathrm{n}^{-1}\right)$ in SRSWOR is accurate to this order and is not available in the past. The expressions for SRSWR can be obtained from these expressions when $\mathrm{N} \rightarrow \infty$ and coincides with the expressions given by Tripathi et al. (2002). The algebra is very lengthy and in the appendix we just illustrate the computation of this. The appendix also contain various moments of sample mean and sample variance in SRSWOR.

Theorem 2.3: Let $\hat{\theta}_{y_{R}}{ }^{(i)}$ and $\hat{\theta}_{\mathrm{y}_{\mathrm{p}}{ }^{(j)}}, \mathrm{i}=1,2,3, \mathrm{j}=1,2,3$ denote the ratio and product estimators of C.V of the study variable when mean, variance and C.V of the auxiliary variable are available for estimation. We list below the bias and MSE expressions to the order of $O\left(\mathrm{n}^{-1}\right)$ in SRSWOR.

2.3.1: Sample C.V.

$$
\hat{\theta}_{y}=\frac{S_{y}}{\bar{y}}
$$

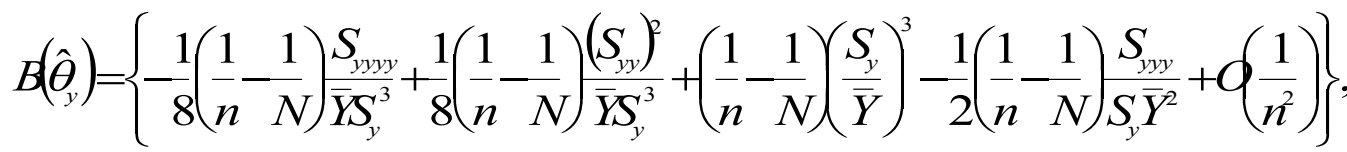

$M\left(\hat{\theta}_{y}\right)=\left\{\frac{1}{4}\left(\frac{1}{n}-\frac{1}{N}\right) \frac{S_{y y y y}}{S_{y}^{2} \bar{Y}^{2}}-\frac{1}{4}\left(\frac{1}{n}-\frac{1}{N}\right) \frac{\left(S_{y y}\right)^{2}}{S_{y}^{2} \bar{Y}^{2}}+\left(\frac{1}{n}-\frac{1}{N}\right)\left(\frac{S_{y}}{\bar{Y}}\right)^{4}-\left(\frac{1}{n}-\frac{1}{N}\right)\left(\frac{S_{y y y}}{\bar{Y}^{3}}\right)+\oint\left(\frac{1}{n^{2}}\right)\right\}$.

\subsection{2: Ratio estimator}

$$
\hat{\theta}_{y_{R}}^{(1)}=\frac{s_{y}}{\bar{y}} \times \frac{\bar{X}}{\overline{\mathrm{x}}} .
$$

$B\left(\hat{\theta}_{y_{R}}^{(1)}\right)=\left\{\begin{array}{l}\left.\left(\frac{1}{n}-\frac{1}{N}\right)\left(\frac{S_{y}}{\bar{Y}}\right)^{3}+\left(\frac{1}{n}-\frac{1}{N}\right) \frac{S_{x}^{2}}{\overline{\bar{Y}}} \frac{S_{y}}{\bar{X}^{2}}-\frac{1}{8}\left(\frac{1}{n}-\frac{1}{N}\right) \frac{S_{y y y}}{S_{y}^{3} \bar{Y}}+\frac{1}{8}\left(\frac{1}{n}-\frac{1}{N}\right) \frac{\left(S_{y}^{2}\right)^{2}}{S_{y}^{3} \bar{Y}}+\left(\frac{1}{n}-\frac{1}{N}\right)\right) \frac{S_{x} S_{y}}{\bar{X} \bar{Y}^{2}} \\ -\frac{1}{2}\left(\frac{1}{n}-\frac{1}{N}\right) \frac{S_{x y y}}{S_{y} \bar{X} \bar{Y}}-\frac{1}{2}\left(\frac{1}{n}-\frac{1}{N}\right) \frac{S_{y y y}}{\bar{Y}^{2} S_{y}}+Q\left(\frac{1}{n^{2}}\right)\end{array}\right\}$

$M\left(\hat{\theta}_{y_{R}}^{(1)}\right)=\left\{\begin{array}{l}\left(\frac{1}{n}-\frac{1}{N}\right)\left(\frac{S_{y}}{\bar{Y}}\right)^{4}+\left(\frac{1}{n} \frac{1}{N}\right) \frac{S_{x}^{2} S_{y}^{2}}{\overline{\bar{Y}}^{2} \bar{X}^{2}}+\frac{1}{4}\left(\frac{1}{n}-\frac{1}{N}\right) \frac{S_{y y y y}}{S_{y}^{S^{2}}}-\frac{1}{4}\left(\frac{1}{n}-\frac{1}{N}\right) \frac{S_{y}^{2}}{\bar{Y}^{2}}+2\left(\frac{1}{n}-\frac{1}{N}\right) \frac{S_{x} S_{y}^{2}}{\bar{Y}^{3} \bar{X}} \\ -\left(\frac{1}{n}-\frac{1}{N}\right) S_{y y y}^{\bar{Y}^{3}}-\left(\frac{1}{n}-\frac{1}{n}\right) \frac{S_{x y y}}{\bar{X} \bar{Y}^{2}}+\phi\left(\frac{1}{n^{2}}\right)\end{array}\right\}$

2.3.3: Ratio estimator.

$$
\hat{\theta}_{y_{R}}^{(2)}=\frac{S_{y}}{\bar{y}} \times \frac{S_{x}^{2}}{S_{x}^{2}}
$$




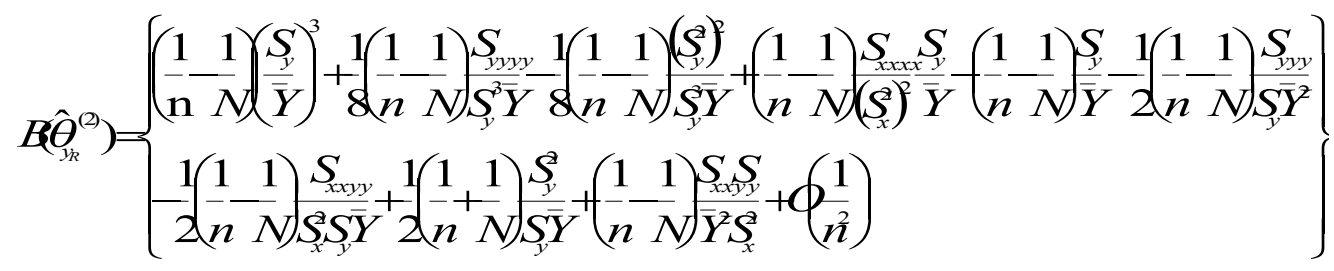

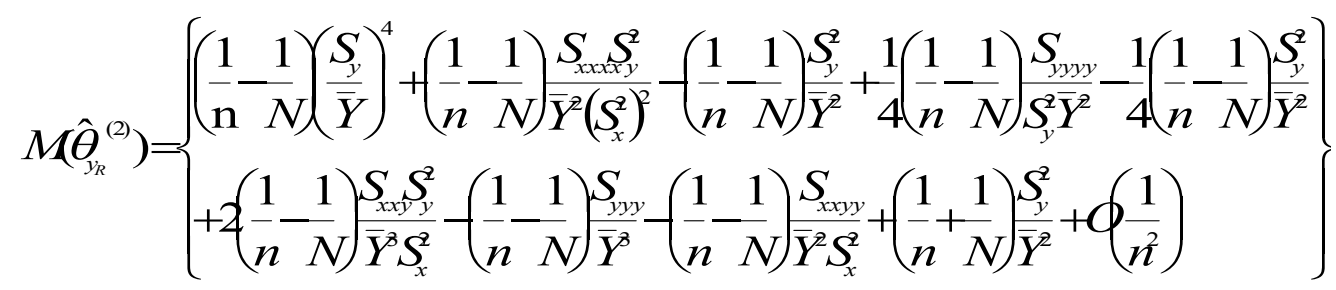

2.3.4: Ratio estimator. $\quad \hat{\theta}_{y_{R}}{ }^{(3)}=\frac{S_{y}}{\bar{y}} \times \frac{S_{x} / \bar{X}}{S_{x} / \bar{x}}$

$$
B\left(\hat{\theta}_{y_{R}}^{(3)}\right)=\left\{\begin{array}{l}
\left(\frac{1}{n}-\frac{1}{N}\right)\left(\frac{S_{y}}{\bar{Y}}\right)^{3}-\frac{1}{8}\left(\frac{1}{n}-\frac{1}{N}\right) \frac{S_{y y y y}}{S_{y}^{S} \bar{Y}}+\frac{1}{8}\left(\frac{1}{n}-\frac{1}{N}\right) \frac{\left(S_{y}^{2}\right)^{2}}{S_{y}^{3} \bar{Y}}+\frac{3}{8}\left(\frac{1}{n}-\frac{1}{N}\right) \frac{S_{x x x} S_{y}}{\left(S_{x}^{2}\right)^{2} \bar{Y}}-\frac{3}{8}\left(\frac{1}{n}-\frac{1}{N}-\frac{1}{N}\right) \frac{S_{y y y}}{S_{y} \bar{Y}^{2}}+\frac{1}{2}\left(\frac{1}{n}-\frac{1}{N}\right) \frac{S_{x x y} S_{y}}{\bar{Y}^{2} S_{x}^{2}}-\left(\frac{1}{n}-\frac{1}{N}\right) \frac{S_{x y} S_{y}}{\bar{Y}^{2} \bar{X}}-\frac{1}{4}\left(\frac{1}{n}-\frac{1}{N}\right) \frac{S_{x x y y}}{S_{y} \bar{Y} S_{x}^{2}}+\frac{1}{4}\left(\frac{1}{n}+\frac{1}{N}\right) \frac{S_{y}^{2}}{S_{y} \bar{Y}} \\
\left.+\frac{1}{2}\left(\frac{1}{n}-\frac{1}{N}\right) \frac{S_{x y y}}{S_{y} \bar{Y} X}-\frac{1}{2}\left(\frac{1}{n}-\frac{1}{N}\right) \frac{S_{x x x} S_{y}}{\bar{Y} S_{x}^{2} \bar{X}}+\mathcal{( \frac { 1 } { n ^ { 2 } }}\right)
\end{array}\right\}
$$

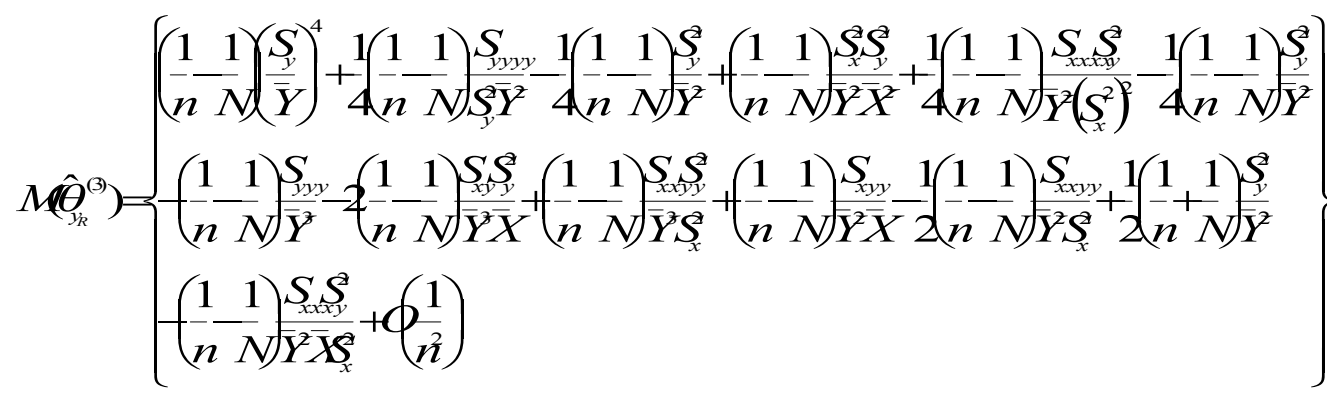

2.3.5: Product estimator. $\quad \hat{\theta}_{y_{P}}{ }^{(1)}=\frac{s_{y}}{\bar{y}} \times \frac{\bar{x}}{\bar{X}}$

$B\left(\hat{\theta}_{y_{p}}^{(1)}\right)=\left\{\begin{array}{l}\left(\frac{1}{\mathrm{n}} \frac{1}{N}\right)\left(\frac{S_{y}}{\bar{Y}}\right)^{3}-\frac{1}{8}\left(\frac{1}{n}-\frac{1}{N}\right) \frac{S_{y y y y}}{S_{y}^{3} \bar{Y}}+\frac{1}{8}\left(\frac{1}{\mathrm{n}}-\frac{1}{N}\right) \frac{\left(S_{y}^{2}\right)^{2}}{S_{y}^{3} \bar{Y}}-\left(\frac{1}{\mathrm{n}}-\frac{1}{N}\right) \frac{S_{x y} S_{y}}{\bar{Y}^{2} \bar{X}}+\frac{1}{2}\left(\frac{1}{\mathrm{n}}-\frac{1}{N}\right) \frac{S_{x y y}}{S_{y} \bar{X} \bar{Y}} \\ \left.-\frac{1}{2}\left(\frac{1}{\mathrm{n}}-\frac{1}{N}\right) \frac{S_{y y y}}{S_{y} \bar{Y}^{2}}+\phi \frac{1}{n^{2}}\right)\end{array}\right\}$

$M\left(\hat{\theta}_{y_{p}}^{\left({ }^{1}\right)}\right)=\left\{\begin{array}{l}\left(\frac{1}{\mathrm{n}} \frac{1}{N}\right)\left(\frac{S_{y}}{\bar{Y}}\right)^{4}+\left(\frac{1}{\mathrm{n}}-\frac{1}{N}\right) \frac{S_{x}^{2} S_{y}^{2}}{\bar{Y}^{2} \bar{X}^{2}}+\frac{1}{4}\left(\frac{1}{\mathrm{n}}-\frac{1}{N}\right) \frac{S_{y y y y}}{S_{y}^{2} \bar{Y}^{2}}-\frac{1}{4}\left(\frac{1}{\mathrm{n}}-\frac{1}{N}\right) \frac{S_{y}^{2}}{\bar{Y}^{2}}-2\left(\frac{1}{\mathrm{n}}-\frac{1}{N}\right) \frac{S_{x y} S_{y}^{2}}{\bar{Y}^{3} \bar{X}} \\ \left(\frac{1}{\mathrm{n}}-\frac{1}{N}\right) S_{\frac{Y_{y y}}{\bar{Y}^{3}}}+\left(\frac{1}{\mathrm{n}}-\frac{1}{N}\right) \frac{S_{x y y}}{\bar{Y}^{2} \bar{X}}+\phi \frac{1}{n^{2}}\end{array}\right\}$ 
2.3.6: Product estimator: $\quad \hat{\theta}_{y_{P}}{ }^{(2)}=\frac{s_{y}}{\bar{y}} \times \frac{s_{x}{ }^{2}}{S_{x}{ }^{2}}$

$B\left(\hat{\theta}_{y_{p}}^{(2)}\right)=\left\{\begin{array}{l}\left(\frac{1}{n}-\frac{1}{N}\right)\left(\frac{S_{y}}{\bar{Y}}\right)^{3}-\frac{1}{8}\left(\frac{1}{n}-\frac{1}{N}\right) \frac{S_{y y y y}}{S_{y}^{3} \bar{Y}}+\frac{1}{8}\left(\frac{1}{n}-\frac{1}{N}\right)\left(\frac{\left.S_{y}^{2}\right)^{2}}{S_{y}^{3} \bar{Y}}-\frac{1}{2}\left(\frac{1}{n}-\frac{1}{N}\right) \frac{S_{y y y}}{S_{y} \bar{Y}^{2}}-\left(\frac{1}{n}-\frac{1}{N}\right) \frac{S_{x x y} S_{y}}{\bar{Y}^{2} S_{x}^{2}}\right. \\ \left.+\frac{1}{2}\left(\frac{1}{n}-\frac{1}{N}\right) \frac{S_{x x y y}}{S_{y} \bar{Y} S_{x}^{2}}-\frac{1}{2}\left(\frac{1}{n}+\frac{1}{N}\right) \frac{S_{x}^{2} S_{y}^{2}}{S_{y} \bar{Y} S_{x}^{2}}+\phi \frac{1}{n^{2}}\right)\end{array}\right\}$

$M\left(\hat{\theta}_{y_{P}}^{(2)}\right)=\left\{\begin{array}{l}\left(\frac{1}{n}-\frac{1}{N}\right)\left(\frac{S_{y}}{\bar{Y}}\right)^{4}+\frac{1}{4}\left(\frac{1}{n}-\frac{1}{N}\right) \frac{S_{y y y y}}{S_{y}^{2} \bar{Y}^{2}}-\frac{1}{4}\left(\frac{1}{n}-\frac{1}{N}\right) \frac{S_{y}^{2}}{\bar{Y}^{2}}+\left(\frac{1}{n}-\frac{1}{N}\right) \frac{S_{x x x x y}}{\bar{Y}^{2}\left(S_{x}^{2}\right)^{2}}-\left(\frac{1}{n}-\frac{1}{N}\right) \frac{S_{y}^{2}}{\bar{Y}^{2}} \\ -\left(\frac{1}{n}-\frac{1}{N}\right) \frac{S_{y y y}}{\bar{Y}^{3}}+\left(\frac{1}{n}-\frac{1}{N}\right) \frac{S_{x x y y}}{\bar{Y}^{2} S_{x}^{2}}-\left(\frac{1}{n}+\frac{1}{N}\right) \frac{S_{y}^{2}}{\bar{Y}^{2}}-2\left(\frac{1}{n}-\frac{1}{N}\right) \frac{S_{x x y} S_{y}^{2}}{\bar{Y}^{3} S_{x}^{2}}+\phi \frac{1}{n^{2}}\end{array}\right\}$

2.3.7: Product estimator.

$$
\hat{\theta}_{y_{P}}{ }^{(3)}=\frac{s_{y}}{\bar{y}} \times \frac{s_{x} / \overline{\bar{x}}}{S_{x} / \bar{X}}
$$

$B\left(\hat{\theta}_{y p}^{(3)}\right)=\left\{\begin{array}{l}\left(\frac{1}{n}-\frac{1}{N}\right)\left(\frac{S_{y}}{\bar{Y}}\right)^{3}+\left(\frac{1}{n}-\frac{1}{N}\right) \frac{S_{x}^{2} S_{y}}{\overline{Y X}^{2}}-\frac{1}{8}\left(\frac{1}{n}-\frac{1}{N}\right) \frac{S_{x x x} S_{y}}{\left(S_{x}^{2}\right)^{2} \bar{Y}}+\frac{1}{8}\left(\frac{1}{n}-\frac{1}{N}\right) \frac{S_{y}}{\bar{Y}}-\frac{1}{8}\left(\frac{1}{n}-\frac{1}{N}\right) \frac{S_{y y y y}}{S_{y}^{3} \bar{Y}} \\ +\frac{1}{8}\left(\frac{1}{n}\right) \frac{\left(S_{y}^{2}\right)^{2}}{S_{y}^{3} \bar{Y}}+\left(\frac{1}{n}-\frac{1}{N}\right) \frac{S_{x y} S_{y}}{\bar{Y}^{2} \bar{X}}-\frac{1}{2}\left(\frac{1}{n}-\frac{1}{N}\right) \frac{S_{y y y}}{S_{y} \bar{Y}^{2}}-\frac{1}{2}\left(\frac{1}{n}-\frac{1}{N}\right) \frac{S_{x x y} S_{y}}{\bar{Y}^{2} S_{x}^{2}}-\frac{1}{2}\left(\frac{1}{n}-\frac{1}{N}\right) \frac{S_{x y y}}{S_{y} \bar{Y} \bar{X}} \\ \left.+\frac{1}{4}\left(\frac{1}{n}-\frac{1}{N}\right) \frac{S_{x x y y}}{S_{y} S_{x}^{2} \bar{Y}}-\frac{1}{4}\left(\frac{1}{n}+\frac{1}{N}\right) \frac{S_{y}^{2}}{S_{y} \bar{Y}}-\frac{1}{2}\left(\frac{1}{n}-\frac{1}{N}\right) \frac{S_{x x x} S_{y}}{\bar{Y} S_{x}^{2} \bar{X}}+Q \frac{1}{n^{2}}\right)\end{array}\right\}$

$$
M\left(\hat{\theta}_{y_{p}}^{(3)}\right)=\left\{\begin{array}{l}
\left.\left(\frac{1}{n}-\frac{1}{N}\right)\left(\frac{S_{y}}{\bar{Y}}\right)^{4}+\frac{1}{4}\left(\frac{1}{n}-\frac{1}{N}\right) \frac{S_{y y y y}}{S_{y}^{2} \bar{Y}^{2}}-\frac{1}{4}\left(\frac{1}{n}-\frac{1}{N}\right) \frac{S_{y}^{2}}{\bar{Y}^{2}}+\frac{1}{4}\left(\frac{1}{n}-\frac{1}{N}\right) \frac{S_{x x x} S_{y}^{2}}{\left(\frac{S_{x}^{2}}{2} \bar{Y}^{2}\right.}-\frac{1}{4}\left(\frac{1}{n}-\frac{1}{N}\right) \frac{S_{x}^{2}}{\bar{Y}^{2}}\right) \\
\frac{1}{\bar{Y}^{2} S_{y}^{2}}-\left(\frac{1}{n}-\frac{1}{N}\right) \frac{S_{y y y}}{\bar{Y}^{3}}-\left(\frac{1}{n}-\frac{1}{N}\right) \frac{S_{x x y} S_{y}^{2}}{S_{x}^{2} \bar{Y}^{3}}+2\left(\frac{1}{n}-\frac{1}{N}\right) \frac{S_{x y} S_{y}^{2}}{\bar{Y}^{3} \bar{X}}+\frac{1}{2}\left(\frac{1}{n}-\frac{1}{N}\right) \frac{S_{x x y y}}{S_{x}^{2} \bar{Y}^{2}} \\
\left.\left.-\frac{1}{2}+\frac{1}{n}\right) \frac{S_{y}^{2}}{\bar{Y}^{2}}-\left(\frac{1}{n}-\frac{1}{N}\right) \frac{S_{x y y}}{\bar{Y}^{2} \bar{X}}-\left(\frac{1}{n}-\frac{1}{N}\right) \frac{S_{x x} S_{y}^{2}}{S_{x}^{2} \bar{Y}^{2} \bar{X}}+Q \frac{1}{n^{2}}\right)
\end{array}\right\}
$$

Proof: The derivations of the expressions are sketched in the Appendix A and B.

\section{Small sample comparison of the estimators.}

Tripathi et al. (2002) compared the asymptotic performance of 22 estimators of C.V with reference to two real life data sets. This includes the seven estimators included in the present investigation. If we confine our attention only to these seven estimators, from their computation it follows that the ratio estimator using the population C.V of the auxiliary variable emerges as the best estimator. Patel and Shah compared the small sample performance of 5 estimators of $\mathrm{C} . \mathrm{V}$ which 
includes the ratio estimator using population C.V of the auxiliary variable and the sample C.V. They compared the small sample performance of the estimators by drawing samples from 19 real life datasets using SRSWOR. The population sizes and sample sizes considered by them are small. In this paper model based approach is used to compare the small sample performance of the seven estimators. The simulation experiment is described below.

\subsection{Simulation Study}

A population of 2000 observations is generated from bivariate normal distribution with parameters $\left(\mu_{x}, \theta_{x}=\frac{\sigma_{x}}{\mu_{x}}\right),\left(\mu_{y}, \theta_{\mathrm{y}}=\frac{\sigma_{y}}{\mu_{y}}\right)$ and $\rho$. These parameters refer to mean, C.V and the correlation co-efficient of the bivariate normal distribution. The value of $\mu_{x}$ and $\theta_{x}$ are first fixed apriori. The value of $\theta_{y}$ is determined such that $\frac{\theta_{y}}{\theta_{x}}$ is equal to $0.5,1.0,1.5$ and 2.0. The correlation co-efficient $\rho$ ranged from -0.9 to 0.9 with an increment of 0.2 and the value ' 0 '. A sample of size ' $n$ ' was selected using SRSWR from the population of ' $N$ ' units. For the sample the value of seven estimators are computed along with the 95\% confidence interval and length of the confidence interval. Using 10,000 replicated samples the bias and MSE of these estimators are also obtained. The small sample estimate of the coverage probability of the confidence interval is obtained by calculating the number of times the true parameter value $\theta_{\mathrm{y}}$ is included in the confidence interval. The average length of the confidence interval is also computed.

\subsection{Results}

The purpose of this simulation is to make an extensive comparison of the performance of the ratio and product estimators in terms of i) MSE ii) Coverage probability of the confidence interval iii) Length of the Confidence Interval. In many of the recent studies, coverage probability and length of the confidence interval is widely used for the comparison of the estimators (see Mahmouvand and Hassani (2007)). Whenever the estimators maintain the nominal coverage probability (1- $\alpha)$ the estimator is considered as the best, if the average length of the confidence interval is the shortest among the various estimators used in the comparison. This conclusion is further strengthened by looking at the small sample MSE of the estimators.

In the present investigation we have taken $\alpha=0.05$ and consider that the confidence level for an estimator is maintained, if the estimated coverage probability $\geq 0.90\left(\approx 5 \%\right.$ error in the coverage probability). For fixed value of $\theta_{\mathrm{y}}$ and $\rho$, we get 4 confidence intervals corresponding to 4 values of $\theta_{x}$. Whenever 
the confidence level is maintained in 3 or more cases (out of 4), the mean of the average length of the confidence interval is also computed and used for comparisons. The values of the $\mathrm{C} . \mathrm{V}$ of the study variable used in the simulations were $0.1,0.3,0.5,0.8,1.0$, and 2.0 . For each fixed value of the study variable, a set of 4 values of $C . V$ of the auxiliary variable are considered. They are $0.5,1.0$, 1.5 and 2 times the C.V of the study variable. The correlation co-efficient used in the simulation study are $-0.9,-0.7,-0.5,-0.3,-0.1,0,0.1,0.3,0.5,0.7,0.9$.The sample sizes considered are $n=100,200$. The confidence level used in the investigation $=0.95$. The total no. of configurations works out to be $6^{*} 4^{*} 11^{*} 2=528$.

The 528 tables regarding the MSE, coverage probability and length of the confidence interval corresponding to the 7 estimators are carefully examined. The results are summarized in the table (3.1) and (3.2). The results are presented in the following table for the sample size $n=100$. The pattern of the results was similar for $n=200$ and hence are not presented.

\begin{tabular}{|c|c|c|c|c|c|c|c|c|c|c|c|c|}
\hline \multirow{2}{*}{\multicolumn{2}{|c|}{$\begin{array}{c}\text { C.V of the } \\
\text { study variable }\end{array}$}} & \multicolumn{11}{|c|}{$\begin{array}{c}\text { Correlation Co-efficient } \\
\text { ' } r \text { ' }(n=100)\end{array}$} \\
\hline & & -0.9 & -0.7 & -0.5 & -0.3 & -0.1 & 0 & 0.1 & 0.3 & 0.5 & 0.7 & 0.9 \\
\hline \multirow{3}{*}{0.1} & $\hat{\theta}_{y}$ & 0.0281 & 0.0287 & 0.0282 & 0.0267 & 0.0270 & 0.0275 & 0.0284 & 0.0266 & 0.0271 & 0.0285 & 0.0278 \\
\hline & $\hat{\theta}_{y_{R}}{ }^{(1)}$ & 0.0279 & 0.0277 & 0.0286 & 0.0270 & 0.0274 & 0.0281 & 0.0294 & 0.0274 & 0.0281 & 0.0279 & 0.0275 \\
\hline & $\hat{\theta}_{y_{R}}{ }^{(3)}$ & 0.0194 & 0.0273 & 0.0357 & 0.0374 & 0.0386 & 0.0395 & 0.0392 & 0.0369 & 0.0333 & 0.0271 & 0.0184 \\
\hline \multirow{3}{*}{0.3} & $\hat{\theta}_{y}$ & 0.0880 & 0.1024 & 0.0865 & 0.0899 & 0.0904 & 0.0884 & 0.0868 & 0.0889 & 0.0883 & 0.0998 & 0.0894 \\
\hline & $\hat{\theta}_{y_{R}}(1)$ & 0.0854 & 0.0898 & 0.0925 & 0.0933 & 0.0997 & 0.1009 & 0.1010 & 0.0998 & 0.1020 & 0.0910 & 0.0878 \\
\hline & $\hat{\theta}_{y_{R}}{ }^{(3)}$ & 0.0835 & 0.0886 & 0.1186 & 0.1242 & 0.1272 & 0.1305 & 0.1283 & 0.1188 & 0.1102 & 0.0870 & 0.0694 \\
\hline \multirow{3}{*}{0.5} & $\hat{\theta}_{y}$ & 0.1742 & 0.1841 & 0.1702 & 0.1692 & 0.1703 & 0.1647 & 0.1646 & 0.1673 & 0.1722 & 0.1894 & 0.1783 \\
\hline & $\hat{\theta}_{y_{R}}{ }^{(1)}$ & 0.1676 & 0.1728 & 0.1860 & 0.1969 & 0.2098 & 0.2102 & 0.2136 & 0.2175 & 0.2314 & 0.1757 & 0.1661 \\
\hline & $\hat{\theta}_{y_{R}}{ }^{(3)}$ & 0.1581 & 0.1632 & 0.2403 & 0.2661 & 0.2594 & 0.2588 & 0.2454 & 0.2348 & 0.2090 & 0.1695 & 0.1422 \\
\hline \multirow{3}{*}{0.8} & $\hat{\theta}_{y}$ & 0.3696 & 0.3714 & 0.3289 & 0.3411 & 0.3449 & 0.3426 & 0.3452 & 0.3312 & 0.3191 & 0.3759 & 0.3605 \\
\hline & $\hat{\theta}_{y_{R}}{ }^{(1)}$ & 0.3230 & 0.3366 & 0.3866 & 0.4013 & 0.4313 & 0.4562 & 0.4815 & 0.5018 & 0.5067 & 0.3294 & 0.2960 \\
\hline & $\hat{\theta}_{y_{R}}{ }^{(3)}$ & 0.2653 & 0.2924 & 0.5081 & 0.5187 & 0.5488 & 0.5329 & 0.5228 & 0.4937 & 0.4069 & 0.2880 & 0.2120 \\
\hline \multirow{3}{*}{1.0} & $\hat{\theta}_{y}$ & 0.4066 & 0.4386 & 0.4695 & 0.5031 & 0.5013 & 0.4963 & 0.4746 & 0.4655 & 0.4682 & 0.4478 & 0.4174 \\
\hline & $\hat{\theta}_{y_{R}}{ }^{(1)}$ & 0.3892 & 0.4313 & 0.5795 & 0.6698 & 0.7041 & 0.7470 & 0.7315 & 0.7649 & 0.7980 & 0.4382 & 0.3922 \\
\hline & $\hat{\theta}_{y_{R}}{ }^{(3)}$ & 0.3687 & 0.4290 & 0.8695 & 0.8562 & 0.8122 & 0.7821 & 0.7702 & 0.6577 & 0.6175 & 0.4302 & 0.3715 \\
\hline \multirow{3}{*}{2.0} & $\hat{\theta}_{y}$ & 1.7090 & 1.8502 & 1.8206 & 1.8441 & 1.9062 & 2.0110 & 1.9486 & 1.7664 & 1.7178 & 1.8111 & 1.7152 \\
\hline & $\hat{\theta}_{y_{R}}{ }^{(1)}$ & 1.2824 & 1.4407 & 1.9976 & 2.3442 & 2.7502 & 3.0210 & 2.9543 & 3.0551 & 3.2256 & 1.4691 & 1.2339 \\
\hline & $\hat{\theta}_{y_{R}}(3)$ & 1.1325 & 1.3597 & 3.0691 & 3.1681 & 2.8189 & 3.0157 & 2.7750 & 2.3664 & 2.0741 & 1.3998 & 1.0824 \\
\hline
\end{tabular}


Table 3.1: Average length of the confidence interval for $\alpha=0.05$

\begin{tabular}{|c|c|c|c|c|c|c|c|c|c|c|c|c|}
\hline \multirow{2}{*}{\multicolumn{2}{|c|}{$\begin{array}{l}\text { C.V of the } \\
\text { study variable }\end{array}$}} & \multicolumn{11}{|c|}{$\begin{array}{l}\text { Correlation Co-efficient ' } r \text { ' } \\
(n=100)\end{array}$} \\
\hline & & -0.9 & -0.7 & -0.5 & -0.3 & -0.1 & 0 & 0.1 & 0.3 & 0.5 & 0.7 & 0.9 \\
\hline \multirow{3}{*}{0.1} & $\hat{\theta}_{y}$ & $\begin{array}{l}0.0532 \\
* 10^{-3}\end{array}$ & $\begin{array}{l}0.0542 \\
* 10^{-3}\end{array}$ & $\begin{array}{l}0.0512 \\
* 10^{-3}\end{array}$ & $\begin{array}{l}0.0457 \\
* 10^{-3}\end{array}$ & $\begin{array}{l}0.0497 \\
* 10^{-3}\end{array}$ & $\begin{array}{c}0.0494 \\
* 10^{-3}\end{array}$ & $\begin{array}{l}0.0517 \\
* 10^{-3}\end{array}$ & $\begin{array}{l}0.0465 \\
* 10^{-3}\end{array}$ & $\begin{array}{l}0.0517 \\
* 10^{-3}\end{array}$ & $\begin{array}{c}0.0534 \\
* 10^{-3}\end{array}$ & $\begin{array}{l}0.0526 \\
* 10^{-3}\end{array}$ \\
\hline & $\hat{\boldsymbol{\theta}}_{y_{R}}$ & 0.0522 & $\begin{array}{l}0.0496 \\
* 10^{-3}\end{array}$ & $\begin{array}{c}0.0538 \\
* 10^{-3}\end{array}$ & $\begin{array}{l}0.0502 \\
* 10^{-3}\end{array}$ & $\begin{array}{l}0.0520 \\
* 10^{-3}\end{array}$ & $\begin{array}{l}0.0527 \\
* 10^{-3}\end{array}$ & $\begin{array}{l}0.0528 \\
* 10^{-3}\end{array}$ & $\begin{array}{l}0.0510 \\
* 10^{-3}\end{array}$ & $\begin{array}{l}0.0557 \\
* 10^{-3}\end{array}$ & $\begin{array}{l}0.0521 \\
* 10^{-3}\end{array}$ & $\begin{array}{l}0.0502 \\
* 10^{-3}\end{array}$ \\
\hline & $\hat{\boldsymbol{\theta}}_{y_{R}}$ & $\begin{array}{l}0.0248 \\
* 10^{-3}\end{array}$ & $\begin{array}{l}0.0489 \\
* 10^{-3}\end{array}$ & $\begin{array}{c}0.0770 \\
* 10^{-3}\end{array}$ & $\begin{array}{l}0.0886 \\
* 10^{-3}\end{array}$ & $\begin{array}{c}0.0963 \\
* 10^{-3}\end{array}$ & $\begin{array}{c}0.0994 \\
* 10^{-3}\end{array}$ & $\begin{array}{c}0.0102 \\
* 10^{-3}\end{array}$ & $\begin{array}{c}0.0891 \\
* 10^{-3}\end{array}$ & $\begin{array}{c}0.0724 \\
* 10^{-3}\end{array}$ & $\begin{array}{l}0.0498 \\
* 10^{-3}\end{array}$ & $\begin{array}{l}0.0199 \\
* 10^{-3}\end{array}$ \\
\hline \multirow{3}{*}{0.3} & $\hat{\theta}_{y}$ & 0.0006 & 0.0008 & 0.0005 & 0.0005 & 0.0006 & 0.0005 & 0.0005 & 0.0006 & 0.0006 & 0.0008 & 0.0007 \\
\hline & $\hat{\boldsymbol{\theta}}_{y_{R}}^{(1)}$ & 0.0005 & 0.0006 & 0.0006 & 0.0007 & 0.0008 & 0.0007 & 0.0009 & 0.0007 & 0.0008 & 0.0007 & 0.0005 \\
\hline & $\hat{\boldsymbol{\theta}}_{y_{R}}$ & 0.0004 & 0.0005 & 0.0008 & 0.0010 & 0.0012 & 0.0011 & 0.0012 & 0.0010 & 0.0009 & 0.0006 & 0.0003 \\
\hline \multirow{3}{*}{0.5} & $\hat{\theta}_{y}$ & 0.0022 & 0.0026 & 0.0019 & 0.0018 & 0.0018 & 0.0019 & 0.0018 & 0.0021 & 0.0022 & 0.0025 & 0.0023 \\
\hline & $\hat{\boldsymbol{\theta}}_{y_{R}}$ & 0.0019 & 0.0020 & 0.0023 & 0.0027 & 0.0025 & 0.0026 & 0.0029 & 0.0033 & 0.0026 & 0.0019 & 0.0018 \\
\hline & $\hat{\boldsymbol{\theta}}_{y_{R}}$ & 0.0017 & 0.0018 & 0.0028 & 0.0038 & 0.0037 & 0.0041 & 0.0038 & 0.0035 & 0.0029 & 0.0018 & 0.0016 \\
\hline \multirow[b]{3}{*}{0.8} & $\hat{\theta}_{y}$ & 0.0083 & 0.0085 & 0.0076 & 0.0073 & 0.0079 & 0.0080 & 0.0082 & 0.0079 & 0.0078 & 0.0086 & 0.0083 \\
\hline & $\hat{\boldsymbol{\theta}}_{y_{R}}^{(1)}$ & 0.0075 & 0.0077 & 0.0079 & 0.0083 & 0.0092 & 0.0096 & 0.0095 & 0.0090 & 0.0087 & 0.0071 & 0.0078 \\
\hline & $\hat{\theta}_{y_{R}}{ }^{(3)}$ & 0.0041 & 0.0057 & 0.0086 & 0.0095 & 0.0105 & 0.0112 & 0.0118 & 0.0099 & 0.0094 & 0.0056 & 0.0048 \\
\hline \multirow{3}{*}{1.0} & $\hat{\theta}_{y}$ & 0.0148 & 0.0152 & 0.0144 & 0.0139 & 0.0146 & 0.0148 & 0.0150 & 0.0143 & 0.0140 & 0.0155 & 0.0146 \\
\hline & $\hat{\boldsymbol{\theta}}_{y_{R}}$ & 0.0124 & 0.0138 & 0.0159 & 0.0164 & 0.0166 & 0.0172 & 0.0178 & 0.0168 & 0.0155 & 0.0133 & 0.0110 \\
\hline & $\hat{\boldsymbol{\theta}}_{y_{R}}^{(3)}$ & 0.0078 & 0.0107 & 0.0162 & 0.0179 & 0.0180 & 0.0192 & 0.0198 & 0.0183 & 0.0172 & 0.0112 & 0.0098 \\
\hline \multirow{3}{*}{2.0} & $\hat{\theta}_{y}$ & 0.1985 & 0.2137 & 0.2019 & 0.2037 & 0.2091 & 0.2100 & 0.2120 & 0.2098 & 0.2017 & 0.2079 & 0.1998 \\
\hline & $\hat{\theta}_{y_{R}}^{(1)}$ & 0.1826 & 0.1889 & 0.1977 & 0.2067 & 0.2098 & 0.2148 & 0.2166 & 0.2125 & 0.2014 & 0.1872 & 0.1818 \\
\hline & $\hat{\boldsymbol{\theta}}_{y_{R}}$ & 0.0996 & 0.1140 & 0.1998 & 0.2160 & 0.2190 & 0.2230 & 0.2236 & 0.2226 & 0.2106 & 0.1236 & 0.1040 \\
\hline
\end{tabular}

Table 3.2: Small sample MSE of the best three estimators

Table (3.1) presents the average length of the confidence interval for the best 3 estimators cross classified by population C.V of the study variable and the correlation co-efficient. Since all the seven estimators (3 ratio, 3 product and sample C.V) maintain the $95 \%$ confidence level, the estimated coverage probabilities are not shown in the table. From the table it is cleat that the ratio estimator using the population C.V and population mean of the auxiliary variable emerges as the best estimator when the study variable and auxiliary variable are 
highly correlated $(|\rho| \geq 0.7)$. When the study and auxiliary variables are moderately or mildly correlated $(|\rho| \leq 0.5)$, the sample C.V emerges as the best estimator followed by the ratio estimator where population mean of the auxiliary variable is used. It may be noted that the performance of the sample C.V does not depend on the correlation co-efficient and the mild fluctuation in the average length of the confidence interval is due to sampling fluctuations. A similar conclusion emerges when we compare the small sample MSE of the seven estimators (see Table (3.2)).

Fig (3.1) and (3.2), represents the average length of the confidence interval for the seven estimators when the population C.V of the study variable is 0.1 and 1.0 indicating the scenario of low and high variability in the study variable. From the figures and the results not reported here it follows that for the ratio estimators using the population C.V or population mean of the auxiliary variable at fixed value of $\mathrm{C} . \mathrm{V}$ of the study variable, the length of the confidence interval is smaller when the study and auxiliary variable are highly correlated. The length of the confidence interval increases when the correlation co-efficient is in the range of 0.5 to 0.5 . For fixed correlation co-efficient the average length of the confidence interval increases as the C.V of the study variable increases for all the estimators.
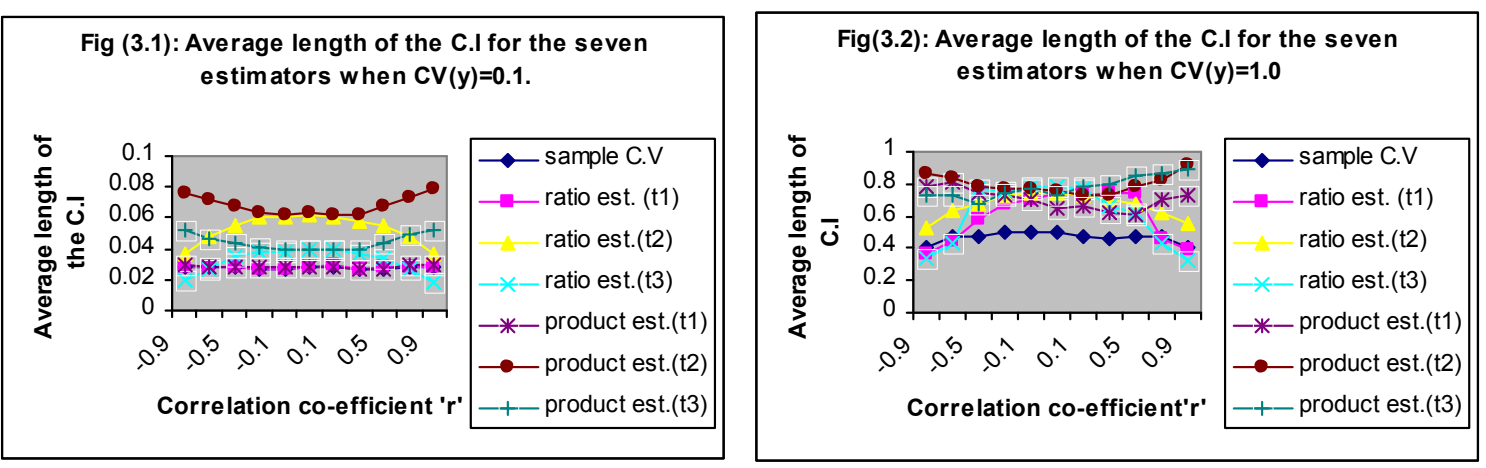

When the study and auxiliary variables are highly correlated the product estimators do not perform well compared to the best ratio estimators and the sample C.V. When the correlation co-efficient is in the range of -0.5 to 0.5 , although some of the product estimators perform better compared to the previous best ratio estimators of $C . V$, their performance is poor compared to the sample C.V. Thus product estimators do not perform well compared to some of the ratio estimators of C.V or the sample C.V.

\section{Discussions}

Tripathi et al. (2002) compared the asymptotic MSE of the 22 estimators including 3 ratio and 3 product type estimators considered in this paper. The conclusion arrived in their paper is that among these seven estimators, the ratio estimator using the information on the population C.V of the auxiliary variable perform better than the sample C.V. The product estimators perform poorly compared to the sample C.V. The population correlation co-efficient for the two 
datasets considered in their paper was 0.9948 and 0.7528 . Patel and Shah (2009) compared the small sample performance of the 5 estimators of C.V which includes the sample C.V and the ratio estimator using population C.V of the auxiliary variable. They used real life datasets for carrying out the simulation. The correlation co-efficient considered in their paper ranges from 0.56 to 1.0. The ratio estimator using the information on the population C.V of the auxiliary variable performs better than the sample C.V in six populations whenever the correlation co-efficient is high or C.V of the study variable is large. Thus from the above two studies and the present investigation it follows that the ratio estimator using population C.V and population mean of the auxiliary variable performs better compared to the sample C.V. In this investigation an attempt is also made to study the impact of the C.V of the auxiliary variable for each fixed value of C.V of the study variable and the correlation co-efficient on the ratio and product estimators. As described in the simulation study, the average length of the confidence interval and the MSE of the 7 estimators are calculated for 4 combinations of C.V of the auxiliary variable which are $0.5,1.0,1.5$ and 2.0 times the C.V of the study variable.

When $\mathrm{CV}(\mathrm{y})_{-}=0.1, \mathrm{r}=0.9$, the 4 values of average length of the confidence interval(over 10,000 simulations) for the ratio estimator (using the information on population C.V) are $(0.0174,0.0177,0.0187,0.0196)$. For the ratio estimator (using the information on population mean) the corresponding average lengths of the confidence interval are $(0.0267,0.0271,0.0280,0.0284)$. Also the 4 values of MSE of these two estimators are $\left(0.0172 * 10^{-3}, 0.0184^{*} 10^{-3}, 0.0217^{*} 10^{-3}\right.$, $\left.0.0226 * 10^{-3}\right)$ and $\left(0.0391 * 10^{-3}, 0.0453^{*} 10^{-3}, 0.0549 * 10^{-3}, 0.0618^{*} 10^{-3}\right)$.

When $C V(y)=0.5, r=0.9$, the corresponding values for the average length of the confidence interval are $(0.1255,0.1352,0.1480,0.1598)$ and $(0.1521,0.1605$, $0.1726,0.1794)$. The 4 values of MSE corresponding to these estimators are $(0.0014,0.0015,0.0017,0.0018)$ and $(0.0016,0.0017,0.0019,0.0021)$

When $\operatorname{CV}(y)=1.0, r=0.9$, the corresponding values for the average length of the confidence interval for the same two ratio estimators are $(0.3292,0.3387$, $0.3866,0.4316)$ and $(0.3558,0.3675,0.3989,0.4467)$. Similarly the corresponding values of MSE of these two ratio estimators are $(0.0079,0.0087$, $0.0099,0.0125)$ and $(0.0088,0.0099,0.0120,0.0134)$.

A similar pattern emerges when $r=-0.9,-0.7,0.7$. A careful examination of the results indicate that when the $C . V$ of the auxiliary variable is larger than the $C . V$ of the study variable, although the average length and MSE of the best two ratio estimators increases it does not affect the relative performance of the estimators. Thus it is clear that these two ratio estimators perform well when the study and auxiliary variable are highly correlated and this performance does not depend on the C.V of the auxiliary variable. This is an important conclusion that emerges from the current investigation. 


\section{Conclusions}

Tripathi et al. (2002) proposed a class of hybrid estimators of C.V of variable of interest. The hybrid estimator is a mixture of regression and ratio estimators. They obtained an optimum estimator belonging to this class. Their conclusion is further supplemented through a simulation study by Patel and Shah (2009). However these investigations do not provide concluding evidence regarding the usage of simple ratio and product estimators of C.V. In the present investigation we have derived accurate expressions for the bias of the ratio and product estimators to the order of $O\left(\mathrm{n}^{-1}\right)$. In this paper model based performance of the ratio and product estimators of $\mathrm{C} . \mathrm{V}$ is assessed using an extensive simulation. The model used for this purpose is a bivariate normal distribution and the finite population is considered as a sample from the infinite population (similar to the Bayesian Inference). The reason for considering a bivariate normal distribution is that in many papers relating to C.V in the case of infinite models assume that the underlying distribution is normal. As noted in Tripathi et al., the conclusion that emerges for the finite models in the case of SRSWR is also applicable to the infinite models and the present conclusions supplement the research work on C.V of a normal distribution.

The correlation co-efficient used in this investigation ranges from -0.9 to 0.9 and 4 values of the $C . V$ of the auxiliary variable are used to compare the performance of the various estimators for a fixed value of $\mathrm{C} . \mathrm{V}$ of the study variable. Attention is paid to compare the performance of the estimators using average length of the confidence interval which provides a more comprehensive comparison of the estimators than is obtained by the MSE. The overall conclusion is that whenever the study variable is highly correlated with the auxiliary variable two of the ratio estimators which use information on population C.V and population mean of the auxiliary variable performs better than the sample C.V. We recommend the usage of the sample C.V when the study variable is not correlated with the auxiliary variable. An applied scientist can check this assumption using sample correlation co-efficient. The ratio and regression type estimators are popular with the investigators in the allied areas like agriculture and this investigation gives a concluding evidence regarding the usage of the ratio estimators. We do not recommend the use of product estimators.

\section{References}

1. Ahmed, S.E (2002): Simultaneous estimation of Co-efficient of Variation. Journal of Statistical Planning and Inference. 104, 31-51.

2. Chaturvedi, A. and Rani, U (1996): Fixed width confidence interval estimation of Inverse co-efficient of variation in normal population. Microelectronics and Reliability, 36, 1305-1308.

3. Das, A.K. and Tripathi, T.P. (1981 a): Sampling strategies for coefficient of variation using knowledge of the mean using an auxiliary character. Tech. Rep. Stat. Math. 5/81. ISI, Calcutta. 
4. Das, A.K. and Tripathi, T.P. (1981 b): A class of estimators for co-efficient of variation using knowledge on coefficient of variation of an auxiliary character. Paper presented at annual conference of Ind. Soc. Agricultural Statistics. Held at New Delhi, India.

5. Fieller, E.C. (1932): A numerical test of the adequacy of A. T. McKay's approximation. J. Roy. Stat. Soc. 95, pp. 699-702.

6. Koyuncu, N. and Kadilar, C. (2010): On the family of estimators of population mean in stratified random sampling, Pak.J.Statist, 26(2), 427-443.

7. Koyuncu, N. and Kadilar, C. (2009). Ratio and product estimators in stratified random sampling. J. Statist. Plann. and Infer., 139(8), 2552-2558.

8. Kadilar, C. and Cingi, H. (2006): Improvement in variance estimation using auxiliary information. Hacettepe Journal of Mathematical Statistics, 35(1), 111-115.

9. Kadilar, C. and Cingi, H. (2005): A new estimator in stratified random sampling. Commun. in Statist.: Theory and Meth., 34(3), 597-602.

10. Kadilar, C. and Cingi, H. (2003): Ratio estimators in stratified sampling, Biometrical Journal, 45(2), 218-225.

11. McKay, A.T. (1932): Distribution of the Co-efficient of variation and the extended't' distribution. J.Royal.Stat.Soc. 95, 696-698.

12. Murthy M.N. (1977): Sampling Theory and Methods, Statistical Publishing Society, Calcutta.

13. Nairy, K.S. and Rao, K.A. (2003): Tests of coefficient of variation in normal population. Communications in Statistics: Simulations and Computation 32 (3), $641-661$.

14. Patel, P. A. and Shah Rina. (2009): A Monte Carlo comparison of some suggested estimators of Co-efficient of variation in finite population. Journal of Statistics sciences, 1(2), 137-147.

15. Prasad, B and Singh, H.P. (1990): Some improved ratio-type estimators of finite population variance in sample surveys. Commun. Statist-theorymeth, 19(3), 1127-1139.

16. Pearson, E.S. (1932): Comparison of A.T. McKay's approximation with experimental sampling results. J. Royal. Stat. Soc. 95, 703-704.

17. Panichkitkosolkul, W. (2009): Improved confidence intervals for a Co-efficient of variation of a normal distribution. Thailand statistician, 7(2), 193-199.

18. Rajyaguru, A. and Gupta, P. (2002): On the estimation of the co-efficient of variation from finite population-I, Model Assisted Statistics and application, 36(2), 145-156.

19. Rajyaguru, A. and Gupta, P. (2006): On the estimation of the co-efficient of variation from finite population-II, Model Assisted Statistics and application, 1(1), 57-66. 
20. Rao, A.K. and Bhatta, A.R.S. (1989): A note on test for Co-efficient of Variation. Calcutta Statistical Association Bulletin, 38, 151-152.

21. Royall, R.M. and Cumberland, W.G. (1981): An empirical study of the ratio estimator and estimators of its variance. Journal of the American statistical Association, 76(373), 66-88.

22. Sen Amartya. (1973): Poverty, Inequality and Unemployment: Some conceptual issues in measurement. Economic and Political Weekly, 8(31/33), 1457-1464.

23. Shabbir, J. and Gupta, S. (2007): On improvement in variance estimation using auxiliary information. Commun. Stat. Theo. And Meth., 36: 2177-2185.

24. Singh, H.P. and Vishwakarma, G.K. (2008): A family of estimators of population means using auxiliary information in stratified sampling. Commun. Statist-theory-meth, 37, 1038-1050.

25. Singh, H.P. and Vishwakarma, G.K. (2006): Combined ratio-product estimator of finite population mean in stratified sampling, Metodologia de Encuestas, 8, 35-44.

26. Singh, H.P. and Tailor, R. (2005): Estimation of finite population mean with known co-efficient of variation of an auxiliary character. Statistica, 75, 301-313.

27. Srivastava, S.K. (1980): A class of estimators using auxiliary information in sample surveys. Canad. J. Statist, 8(2), 253-254.

28. Srivenkataramana, T., (1980): "A dual to ratio estimator in sample surveys" Biometrika, 67(1), 199-204.

29. Tripathi, T.P. Singh, H.P. and Upadhyaya, L.N. (2002): A general method of estimation and its application to the estimation of co-efficient of variation. Statistics in Transition, 5(6), 887-909.

30. Upadhyaya, L.N., Singh, H.P. and Singh, S. (2004): A class of estimators for estimating the variance of the ratio estimator. J. Japan Statist.Soc.34 (1), 47-63.

31. Verril, S. (2003): Confidence bounds for normal and log-normal distribution co-efficient of variation, Research paper, EPL-RP-609, Madison, Wisconsin, US.

\section{Acknowledgement}

This paper is part of the first authors Ph.D. work under the guidance of second author. We are thankful to the anonymous referees for bringing our attention to some salient works on C.V. Their valuable comments have drastically improved the quality of the present paper. 


\section{Appendix A}

To illustrate the derivation of bias and MSE of the ratio/product estimators, we consider the Taylor series expansion of ratio estimator $\hat{\theta}_{y_{R}}{ }^{(3)}$ where information on population C.V of the auxiliary variable is used.

$$
\begin{aligned}
& \text { Case (i): } \hat{\theta}_{y_{R}}{ }^{(3)}=\frac{S_{y}}{\bar{y}} \times \frac{S_{x} / \overline{\bar{X}}}{S_{x} / \bar{x}} \\
& \left(\hat{\theta}_{y_{R}}^{(3)}-\theta_{y_{R}}^{(3)}\right)=\left\{(\bar{y}-\bar{Y}) \frac{S_{\bar{y}}}{\overline{Y^{2}}}+(\bar{x}-\bar{X}) \frac{S_{y}}{\bar{X}}+\left(S_{y}^{2}-S_{y}^{2}\right) \frac{1}{2 S_{y} \bar{Y}}-\left(s_{x}^{2}-S_{x}^{2}\right) \frac{S_{y}}{2 S_{x}^{2}}+(\bar{y}-\bar{Y})^{2} \frac{S_{y}}{\bar{Y}^{3}}-\left(s_{y}^{2}-S_{y}^{2}\right)^{2} \frac{1}{8 S_{y}^{3} \bar{Y}}\right. \\
& +\left(s_{x}^{2}-S_{x}^{2}\right)^{2} \frac{3 S_{y}}{\delta\left(S_{x}^{2}\right)^{2} \bar{Y}}-(\bar{y}-\bar{Y})\left(s_{y}^{2}-S_{y}^{2}\right) \frac{1}{2 S_{y} \bar{Y}^{z}}-(\bar{x}-\bar{X})\left(S_{x}^{2}-S_{x}^{2}\right) \frac{S_{y}}{2 \bar{X} S_{x}^{2}}+(\bar{y}-\bar{Y})\left(S_{x}^{2}-S_{x}^{2}\right) \frac{S_{y}}{2 \bar{Y}^{2} S_{x}} \\
& \left.-(\bar{y}-\bar{Y})(x-\bar{X}) \frac{S_{y}}{\bar{Y}^{2} \bar{X}}-\left(s_{x}^{2}-S_{x}^{2}\right)\left(s_{y}^{2}-S_{y}^{2}\right) \frac{1}{4 S_{y} \bar{Y} S_{x}^{2}}+\left(s_{y}^{2}-S_{y}^{2}\right)(\bar{x}-\bar{X}) \frac{1}{2 S_{y} \bar{X}}\right\}
\end{aligned}
$$

In a similar manner the Taylor series expansion of other ratio/product estimators can be obtained to the order of $O\left(\mathrm{n}^{-1}\right)$. Now to find the bias and MSE of the ratio/product estimator, we require the following moments of sample mean and sample variance under SRSWOR.

\section{Appendix B}

Population Moments in SRSWOR

$$
\begin{aligned}
& E(\bar{x}-\bar{X})=0 \\
& E(\bar{y}-\bar{Y})=0 \\
& E(\bar{x}-\bar{X})^{2}=\left(\frac{1}{n}-\frac{1}{N}\right) S_{x x} \\
& E(\bar{y}-\bar{Y})^{2}=\left(\frac{1}{n}-\frac{1}{N}\right) S_{y y} \\
& E(\bar{x}-\bar{X})(\bar{y}-\bar{Y})=\left(\frac{1}{n}-\frac{1}{N}\right) S_{x y} \\
& E\left(s_{x}{ }^{2}-S_{x}{ }^{2}\right)=0 \\
& E\left(s_{y}{ }^{2}-S_{y}{ }^{2}\right)=0
\end{aligned}
$$




$$
\begin{gathered}
E\left(s_{y}{ }^{2}-S_{y}{ }^{2}\right)^{2}=\frac{(N-n)}{N n} S_{y y y y}-\frac{(N-n)}{N n}\left(S_{y y}\right)^{2}+O\left(\frac{1}{n}\right) \\
E\left(s_{x}{ }^{2}-S_{x}{ }^{2}\right)^{2}=\frac{(N-n)}{N n} S_{x x x x}-\frac{(N-n)}{N n}\left(S_{x x}\right)^{2}+O\left(\frac{1}{n}\right) \\
E\left({s_{x}}^{2}-S_{x}{ }^{2}\right)^{2}(\bar{X}-\bar{X})=\frac{N-n}{N n} S_{x x x}+O\left(\frac{1}{n}\right) \\
E\left(s_{x}{ }^{2}-S_{x}{ }^{2}\right)(\bar{y}-\bar{Y})=\frac{N-n}{N n} S_{x x y}+O\left(\frac{1}{n}\right) \\
E\left(s_{y}{ }^{2}-S_{y}{ }^{2}\right)(\bar{x}-\bar{X})=\frac{N-n}{N n} S_{x y y}+O\left(\frac{1}{n}\right) \\
E\left(s_{x}{ }^{2}-S_{x}{ }^{2}\right)\left(s_{y}{ }^{2}-S_{y}{ }^{2}\right)=\left(\frac{1}{n}-\frac{1}{N}\right) S_{x x y y}-\left(\frac{1}{n}+\frac{1}{N}\right) S_{x x} S_{y y}+O\left(\frac{1}{n}\right) \\
\text { Note : } S_{y y}=\frac{1}{(N-1)} \sum_{i=1}^{N}\left(Y_{i}-\bar{Y}\right)^{2} \quad S_{x y}=\frac{1}{(N-1)} \sum_{i=1}^{N}\left(X_{i}-\bar{X}\right)\left(Y_{i}-\bar{Y}\right) \\
\mathrm{S}_{x y y}=\frac{1}{(N-1)} \sum_{\mathrm{i}=1}^{\mathrm{N}}\left(\mathrm{X}{ }_{\mathrm{i}}-\overline{\mathrm{X}}\right)\left(Y_{\mathrm{i}}-\bar{Y}\right)^{2} \quad S_{x x y y}=\frac{1}{(\mathrm{~N}-1)} \sum_{i=1}^{N}\left(X_{i}-\bar{X}\right)^{2}\left(Y_{i}-\bar{Y}\right)^{2} \\
S_{y y y y}=\frac{1}{N-1} \sum_{i=1}^{N}\left(Y_{i}-\bar{Y}\right)^{4} \sum_{i=1}^{N}\left(Y_{i}-\bar{Y}\right)^{3}
\end{gathered}
$$

In the similar manner the other moments are defined. 